\title{
Intelectualidad, raza y género: mujeres en afro- rresistencia del valle de Azapa y Arica, Chile ${ }^{1}$
}

\author{
INTELLECTUALITY, RACE AND GENDER: WOMEN IN AFRO-RESISTANCE \\ from the Azapa and Arica valley, Chile
}

Nicole Chávez González

Universidad Alberto Hurtado, Santiago de Chile nicolechg88@gmail.com

RESUMEN: Este artículo entrega una visión antropológica general sobre la comunidad "afrochilena" del norte del país, un pueblo que ha sido alterado por múltiples procesos históricos que repercuten en sus formas de habitar el territorio. Primero, y a través de revisiones bibliográficas, se hace un breve repaso al contexto histórico: esclavitud, conformación de los Estados-nación, Guerra del Pacífico y posterior proceso de chilenización hasta principios de los 2000, donde renace el movimiento afrochileno en el valle de Azapa y Arica, Chile. Luego, gracias a la experiencia etnográfica y trabajo en terreno, se reivindican y ponen en valor los roles intelectuales de las mujeres y su adscripción racial en la sociedad, por medio de los diferentes papeles que encarnan en pos de la reconstrucción y resistencia de la identidad afrochilena, desde distintos espacios: luchas políticas, traspaso de conocimientos y tradiciones, producciones intelectuales, entre otras. Finalmente, se sostiene la relevancia social, cultural, económica y política

1 El presente artículo encuentra su base en mi tesis de licenciatura de Antropología, UAHC, denominada Mujeres afrodescendientes chilenas, raices africanas en el Valle de Azapa y Arica (2016). 
que tienen dentro de la persistencia, etnogénesis y autorreconocimiento de su comunidad desde la intelectualidad.

Palabras Clave: mujeres afrochilenas, intelectualidad afro, género, Arica y valle de Azapa.

АвsтRAст: This article provides a general anthropological vision of the "Afro-Chilean" community in the north of the country, a people that has been altered by multiple historical processes that had an impact on their ways of inhabiting the territory. First, and through bibliographic reviews, a brief review of the historical context is made: slavery, the formation of nation-states, the Pacific War and the subsequent Chileanization process, until the early 2000s, when the Afro-Chilean movement was reborn in Arica and the Azapa valley, Chile. Then, thanks to ethnographic experience and field work, the intellectual roles of women and their racial ascription in society are vindicated and valued, through different roles that they embody in pursuit of the reconstruction and resistance of the Afro-Chilean identity, from different spaces: political struggles, knowledge and traditions transfer, intellectual productions, among others. Finally, the social, cultural, economic, and political relevance that they have within the persistence, ethnogenesis and self-recognition of their community is upheld from the intellectually.

Keywords: Afro-Chilean women, Afro-intellectuals, gender, Arica and the Azapa Valley.

\section{INTRODUCCIÓN}

Este artículo se enmarca en los estudios afrolatinoamericanos que De la Fuente y Reid Andrews describen "como el estudio de la población de ascendencia africana en América Latina y, en segundo lugar, como el estudio de las sociedades en las que vive dicha población" (11). Para ello, se investigan las culturas, historias, estrategias y luchas de las y los negros en la región, entendiendo la raza como una categoría de diferencia, estratificación y desigualdad que son claves en los procesos de formación nacional. De esta manera, en los últimos veinte años, los estudios afro en América Latina -y en particular sobre los afrochilenos- han tenido un crecimiento visible, con nuevas investigaciones y perspectivas teóricas. Una de estas nuevas 
perspectivas tiene como objetivo enfocarse en las y los afrodescendientes y sus roles en las sociedades latinoamericanas desde sus propias elaboraciones intelectuales (Arre y Barrenechea; Oliva, "Intelectuales").

Estas personas son descendientes de una trata transatlántica que, de manera forzada, hizo migrar a millones de africanos esclavizados. Este proceso violó los derechos humanos de una población entera, obligada a desempeñarse en múltiples labores como transporte terrestre y marítimo, minería, milicia, agricultura y, principalmente, servidumbre (Vial 24; Mellafe 14). Específicamente en el valle de Azapa, las y los africanos trabajaron en la producción de algodón, caña de azúcar y olivos, junto con los servicios en hogares. Estas labores recaían en la población femenina y aumentaron durante el último siglo de la colonia, puesto que la tenencia de mano de obra esclavizada era símbolo de poder y riqueza (Vidal 5).

Estas identidades afrodescendientes se han racializado desde la colonización hasta la actualidad, tiempo durante el cual se les clasificó en posiciones binarias que se basaban en términos de blanco y no-blanco, poseyendo la población blanca un estatuto superior y segregando a quienes no pertenecieran a esta categoría desde una desigualdad estructural (Fanon 14; Curiel, "Género" 15). De esta manera, se destaca el hecho de que en procesos políticos hay más de una condición de diferencia y aquí las examinamos en sus múltiples niveles de análisis y de interacciones entre los mismos, relevando la falta de interés en los temas de género de los movimientos e intelectualidades negras en diferentes países de nuestro continente, desde la colonia hasta la actualidad (Guridy y Hooker 4).

Actualmente, gran parte de la población que se autorreconoce como afrochilena habita en la Región de Arica y Parinacota ${ }^{2}$, en el extremo norte del país. Este es un territorio en que se mezclan diferentes identidades, pues es una zona donde un $35,7 \%$ se reconoce como indígena, junto a un $8 \%$ de migrantes que responden al carácter fronterizo de la región (INE, Sintesis 16). Estas cifras convierten a esta área en un mosaico cultural. Según la "Encuesta de Caracterización Afrodescendiente" realizada por el Instituto Nacional de Estadística

2 La Región de Arica y Parinacota se encuentra entre los $17^{\circ} 30^{\prime}$ y los $21^{\circ} 28^{\prime}$ de latitud sur aproximadamente, limita en el norte con la República del Perú, al sur con la región de Tarapacá, en su lado este con la República de Bolivia y al oeste con el Océano Pacífico. 
(INE) el año 2013 , un 4,7\% $\%^{3}$ de la población regional se autodefine como afrochileno, lo que equivale a 8.415 habitantes sobre un total de 226.068 personas que viven en la región (INE, Sintesis 5). La comunidad reside principalmente en la ciudad urbana de Arica y en el sector rural del valle de Azapa, ubicado a unos $5 \mathrm{~km}$ hacia el interior ${ }^{4}$.

Existe una reinvención cultural de las comunidades afrodescendientes a través de una etnogénesis ${ }^{5}$, con un discurso eficaz que se hace cada día más masivo y reconocido, lo que las incorpora como actores y agentes sociales y políticos en América Latina (Bengoa 29). La demanda principal del movimiento afrochileno es la "dignidad de trato"; es decir, la no discriminación y el reconocimiento legal tanto del Estado como de la sociedad civil para llegar a un nuevo pacto. Una de las principales causas/ consecuencias de la trata transatlántica, el esclavismo, la chilenización y posterior invisibilización es el racismo y sus diferentes discriminaciones, como fenómenos interrelacionados que permiten entender las rupturas y continuidades en las identidades afrodescendientes.

Luego de casi veinte años de lucha, el 7 de marzo del 2019 se aprobó la Ley de Reconocimiento del Pueblo Tribal Afrodescendiente ${ }^{6}$, una instancia que se gestó desde las mismas bases por líderes de la comunidad, con el apoyo de diputados y senadores de la región. Esta ley establece la obligación del Estado a valorar la cultura afrochilena y les reconoce como parte del patrimonio cultural inmaterial de Chile; además, promueve una reparación histórica a través del sistema educacional, con unidades

3 Esta cifra es antigua y hasta la actualidad no existen datos nuevos. Con la nueva Ley de Reconocimiento del Pueblo Tribal Afrodescendiente, este hecho podría cambiar, al ser incluidos en el próximo censo a realizarse en el país, junto con saber datos de otras regiones a lo largo de Chile.

4 Su principal poblado es San Miguel de Azapa. Destaca la agricultura y fecundidad de sus tierras, junto a la diversidad cultural: la ancestral cultura chinchorro, indígenas aymara y quechua, alta población afrodescendiente chilena y migrantes de Bolivia.

5 Según Bengoa (2000), la etnogénesis hace referencia a un proceso de reinvención cultural de un grupo determinado, el cual hace uso de préstamos culturales para construir sus propias identidades colectivas (68).

6 "Ley 21151. Otorga reconocimiento legal al pueblo tribal afrodescendiente chileno”. Disponible en https://www.leychile.cl/Navegar?idNorma=1130641 
programáticas abocadas a enseñar sobre esta comunidad. Una de las implicancias más relevantes de esta ley es que estas comunidades serán incluidas en los siguientes censos de población.

En términos metodológicos, este artículo se basa en las interrogantes surgidas en diferentes investigaciones que se han desarrollado, desde mayo del 2014 hasta la fecha, con afrodescendientes del territorio nortino. El primer terreno se enmarcó en el "Proyecto de Recolección y Clasificación de Datos etnoterritoriales", un estudio pionero en cuanto a la recopilación de características culturales de la comunidad en pos de implementar políticas públicas relevantes y apoyar en procesos legales a esta población (Campos y Espinosa 6). También se han realizado revisiones historiográficas de investigaciones, archivos y documentos, a la par de un trabajo cualitativo enfocado en la etnografía y la participación observante en sus vidas y celebraciones (Hammersley y Atkinsons 16; Guber 60), además de múltiples entrevistas y relatos de vida.

En particular, en esta investigación se identifican los papeles y roles intelectuales de la población afrofemenina en la reconstrucción de la memoria histórica y las dinámicas de identidad. Además, se describen e identifican sus roles participativos y sus papeles como lideresas en el proceso de etnogénesis, junto a las producciones intelectuales que han realizado. Existen diversas formas en que se expresan las políticas de identidades e intelectualidades de las afrochilenas. En algunos espacios, se conservan y recrean elementos de la herencia y memoria de la cultura africana junto a mixturas interculturales, que se han entretejido con el paso de las décadas y los trabajos intelectuales que han desarrollado. Así, se entiende que la "función de articular ideas, reflexiones y conceptos [...] a su vez, es necesari[a] [para] comprenderlos como sujetos que ejercen la función intelectual de manera situada [...] a partir del reconocimiento" (Oliva, "Intelectuales" 54).

7 Proyecto Fondecyt 1150876, Proyecto Fondap CIIR, "Proyecto Recoleción y Clasificación de Datos etnoterritoriales afrodescendientes de la Región de Arica y Pariancota. Apoyado por la Universidad Academia de Humanismo Cristiano y la Municipalidad de Arica". 


\section{Afrodescendencia, INTEleCtUAlidAd y GÉNERo}

En cuanto a lo teórico, esta investigación busca resaltar la categoría de dinámicas de identidades, género y la interseccionalidad transversal entre etnicidad ${ }^{8}$, raza y clase9 (Hill Collins 255; Davis 72; Curiel, "Género" 18), donde patrones de desigualdad racial, de género y de clase se intersecan de tal manera que producen categorías difíciles de desterrar en cuanto a exclusión y sometimiento (Reid Andrews 5). Es importante destacar que "las razas son construcciones sociales. La idea de raza es justo eso: una idea" (Wade 21). No habría, entonces, características físicas definibles, tampoco existen superioridades de unas sobre otras; es un concepto socialmente construido como resultado de procesos históricos que tienen su raíz en la colonización, pero eso no quita su real existencia y las consecuencias que trae consigo. En la actualidad, el racismo se presenta a través de prácticas, dinámicas y discursos que lo avalan, donde sí se considera que una raza es superior y por tanto puede dominar a la otra. Así ha funcionado históricamente y trae consigo usos de discriminaciones académicas, populares y políticas, como en situaciones e interacciones cotidianas (Tijoux 2).

Según Reid Andrews, las identidades raciales continuamente cambian, se manipulan, son dinámicas, se construyen y se instrumentalizan. Hay elementos que desaparecen y otros que se transforman, incluso algunos que se crean e inventan. Así, las dinámicas de las identidades afro son fluidas, relacionales y situacionales, e interactúan con otras formas de identidades y categorías sociales. Aquí sobresale la memoria y expresión cultural propia, con una tradición e historia oral que se erige como la gran escuela de la vida, religión, historia, diversión, entre otras expresiones, sobre procesos y productos de la comunidad (De Friedemann 21). En este sentido, la comunidad afrochilena proclama una cultura basada en conocimientos y valores compartidos sobre ciertas tradicio-

8 La etnicidad se podría definir como una construcción social de identificación de la diferencia y la igualdad, su especificidad va en las diferencias culturales; mientras que la raza serían diferencias fenotípicas (Wade 24). De todos modos, la etnicidad "se vuelve una insatisfactoria categoría analística residual: incluye todas esas formas de categorización cultural donde no existe otro discurso primario de diferenciación, tal como la riqueza, el sexo, la edad, el fenotipo" (Wade 25).

9 La "raza”, igual que el sexo, clase, género, entre otros, han sido concebidas como categorías sociales de poder basadas en ideologías de la diferencia. 
nes orales y su reconstrucción histórica, que van desde sus comidas, música y bailes, a celebraciones de fiestas religiosas como la de la Cruz de Mayo, la del Patrono de San Miguel de Azapa, la de la Virgen de las Peñas, la Pascua de Negros, el No Carnavalón, entre otras (Espinosa "Reconstrucción" 110; Chávez 69; Araya, Chávez y Alarcón 5). Estas prácticas no remiten exclusivamente a un pasado ancestral africano, sino más bien a un sincretismo entre culturas a las que esta comunidad ha dotado de pertenencias, particularidades y territorialidades.

Por otra parte, este artículo emplaza la importancia del Black Feminism [feminismo negro] que surge en Estados Unidos ${ }^{10}$, como también los feminismos afrolatinoamericanos y el caso chileno, pues estas perspectivas "dejan al descubierto las luchas intersectoriales de las mujeres negras en América Latina y que abren nuevas avenidas en el pensamiento afrolatinoamericano" (Guridy y Hooker 248). Los movimientos feministas negros llevan a cabo una lucha política a nivel internacional frente al racismo y sexismo que existe sobre ellas. Estas mujeres asumen que ser de color es más que una definición biológica, sino que una categoría política.

Las mujeres afro critican la universalidad del feminismo occidental, pues este olvida analizar los diferentes contextos-situaciones de cada mujer y sus historias, lo que desemboca en un racismo desde las propias feministas para/con aquellas que viven otras realidades, atravesadas por la etnicidad, raza y clase (Davis 75), junto a una construcción de estereotipos y roles sexualizados y racializados a los que han sido sometidas (Curiel, "Los aportes" 2). Si bien sufren de violencia en lo privado, las feministas negras se enfocan en el ámbito público y la violencia racista histórica y estructural que viene desde los gobiernos e instituciones, debido a la aceptación y promoción de estigmas como también por la condición social ligada a la pobreza y las consecuencias de ello. De este modo, buscan una transformación social a partir de sus experiencias históricas y, por ello, participan en diferentes colectivos feministas antirracistas, en los que realizan acciones y prácticas para tener una visibilidad política y concientizar su protesta, con el racismo como eje negros, feministas y la militancia obrera, que pedían reformas sociales. Por ende, ya no era posible sostener las tipologías raciales. Desde allí la relevancia del feminismo negro y su lucha intelectual y política. 
articulador sobre las relaciones de género sufridas por mujeres negras (Carneiro 2). Finalmente, Carneiro sostiene que el feminismo negro responde a experiencias e ideas que aúnan a las mujeres afrolatinas, a través de: a) el legado de una historia de lucha, b) la interconexión de variables de raza, género y clase, y c) la resistencia hacia los estereotipos (5).

En este artículo, sostenemos que estas variantes impulsan a las intelectuales afro, entendidas tal como señala María Elena Oliva, a una "conceptualización anclada en la toma de conciencia de una experiencia histórica particular [...] El reconocimiento explícito de la identidad negra o afrodescendiente, junto con la problematización de ésta, es un requisito fundamental [...] que remite a un vínculo histórico y político" ("Intelectuales" 58). Lo político, la raza y el género contribuyen a la intelectualidad oral y escrita de este grupo de mujeres afrochilenas.

\section{CONTEXTO HISTÓRICO Y ROLES FEMENINOS}

Desde la esclavitud ${ }^{11}$, las negras se vieron doblemente vulneradas por el hecho de ser mujeres, pues sufrieron explotaciones laborales junto a violaciones físicas reiteradas, que daban respuesta al exotismo que imperaba y al poder que tenían los patrones para subordinarlas. Rosa Soto sostiene que las esclavas eran vientres generadores de mano de obra, mientras que las y los hijos se convertían en bienes que eran transados como negocio (4). De este modo, sus cuerpos se transformaron en mercancía sexual y reproductora del patrimonio esclavista ${ }^{12}$. Por otro lado, y aun cuando desconocemos gran parte de las experiencias e intelectualidades de las esclavas, Viviana Briones rescata el rol de la mujer en la reiterada petición de libertad. Es decir, la manumisión de su progenie a pesar de que esa condición era heredada por la línea materna (11). Durante el siglo XVIII, estas mujeres enfrentaron de manera directa e indirecta al sistema político, judicial y social en el corregimiento de Arica. De este modo, observamos que "aun cuando 'actuaran dentro

11 Si bien los estudios actualmente van en aumento, los diferentes roles de las mujeres negras esclavas en Chile continúan siendo poco abordados.

12 En la zona estudiada, los relatos de las y los colaboradores sostienen la existencia de criaderos de negros en el valle de Lluta, donde un negro semental violaba a esclavas que parían como si fuese animales de ganado. 
del sistema' [...] cuestionaban y desafiaban los propios cimientos sobre los que este descansaba" (Guridy y Hooker 227). Estas estrategias de autoemancipación se constituyen como un espacio de liberación negra en cuanto al pensamiento afrolatino y, en el presente caso, la población femenina adquiere una particular relevancia.

Una vez instauradas las repúblicas, las y los negros se enfrentaron a los procesos nacionales, donde los pensadores afro "lucharon por tener un lugar en las nuevas naciones de las que ahora formaban parte y a las que, en muchos casos, habían ayudado a fundar" (Guridy y Hooker 222), pero en las que aún no eran reconocidos como libres (Vial 158). Las comunidades negras comenzaron a crear instituciones intelectuales y culturales que se establecieron como espacios de dispersión, de discusión y movilización sociopolítica. Por ejemplo, las "ligas de estudiantes” y los "círculos de peruanos", en las que participaban tanto hombres como mujeres y que funcionaban en la zona como "estrategias colectivas, basadas en lazos solidarios, para mantener viva la cultura" (Alarcón, Araya y Chávez 101). Pero, tal como seńalan Guridy y Hooker, este tipo de activismo no pudo desafiar el poder de las nuevas naciones y sus luchas ideológicas de inclusión racial, además de la implantación de un liberalismo e imperialismo apoyado en el racismo científico de la época, que perduró incluso después de la abolición mundial de la esclavitud (221).

Las tierras de Arica y Tacna pertenecieron al Perú hasta la Guerra del Pacífico (1879-1883), momento en que estos territorios fueron anexados a Chile, a la espera de un plebiscito que nunca se realizó. Aquel periodo de incertidumbre fue conocido como "chilenización" y duró de 1884 a 1929. Durante ese tiempo, la mayoría de la población peruana -indígena y negra- sufrió maltratos y asesinatos (Artal 7; Alarcón, Araya y Chávez 8). El aparato estatal impuso una nueva identidad chilena y una xenofobia exacerbada, a través de diferentes maniobras de control y aparatos simbólicos (Salgado, Afrochilenos 164). Según los relatos, las mujeres tuvieron roles importantes en esta época, enfocados en sus papeles como sustentadoras del hogar en cuanto a lo emocional y económico, mientras los hombres iban a guerras o eran asesinados. Estas persecuciones, muertes, malos tratos, despojos y desplazamientos forzados generaron traumas en la población, una marca que se extendió sobre el territorio ariqueño y un nuevo Chile idealizado sin negritud. 
La chilenización funcionó como una "segunda colonización" (Alarcón, Araya y Chávez 100), en la que la comunidad negra escondió su identidad para que su linaje no conociera la historia de un pasado esclavo, peruanizado y chilenizado. Hubo dos características visibles que afectaron a la comunidad negra: lo físico y lo cultural. En sus discursos, se revela la estrategia de blanquear su piel y cambiar rasgos para "mejorar la raza" al "mezclarse" 13 con blancos. Mientras que las costumbres se encapsularon en la memoria de los más antiguos y quedaron en el núcleo íntimo familiar. En paralelo, desaparecen en los censos las categorías raciales y, por ende, de las cifras oficiales nacionales, otra forma de asimilación e inclusión racial por parte del Estado de Chile, que era incluso peor, pues invisibiliza lo más tangible (De la Fuente y Reid Andrews 13).

De este modo, y durante las siguientes décadas la negritud se sigue borrando. Más aún durante la dictadura de Pinochet, quien continúo con lineamientos de asimilar la diversidad cultural en el país bajo el lema de "linaje blanco y raza chilena" (Vidal 2). Durante los noventa, en América Latina resurgen los movimientos indígenas y también afrodescendientes $^{14}$, los que rechazaron las estrategias asimilacionistas y "buscaron en las raíces africanas y las rutas de la diáspora afrolatinoamericana, las anclas de una identidad negra específica que se diferenciaba de los imaginarios dominantes nacionales de cada país" (Guridy y Hooker 248). En este contexto, y de a poco, surge el movimiento afrochileno, junto a la aparición de intelectuales afrochilenos.

Al comienzo, este corpus del pensamiento posee un carácter mayoritariamente femenino, distinto al resto de los países latinoamericanos (Chávez y Araya 9). El año 2000, en Santiago de Chile, se reunieron diferentes redes y organizaciones sociales para realizar la Preconferencia de Durban. Los relatos señalan que se invita a esta reunión a Sonia Salgado, en ese entonces alcaldesa de Camarones ${ }^{15}$, quien asiste con su hermana Marta y una comitiva de personas que poseían características físicas que ponían en

13 Se deja entre comillas porque son dichos de las y los participantes.

14 Concepto que reemplazó del término negro y se convirtió en una categoría política y jurídica para insertarse en la esfera pública, política e internacional. La construcción externa del concepto afrodescendiente, fue el motor primordial para la conformación del movimiento en Chile y ha sido un aporte en cuanto a lograr que la población se auto reconozca como tal (Espinosa, "Afrochilenos" 177; Campos 20).

15 Camarones es una comuna que pertenece a la provincia de Arica. 
duda la blanquitud chilena. La comunidad recuerda la actitud que tuvo el presidente de la República, Ricardo Lagos, quien negó la existencia de negros en Chile. Es ahí donde ellas se paran y gritan: “Existimos los negros, nosotros somos negros!”, un hecho icónico que potencia el discurso de pertenencia y autorreconocimiento (Salgado, "Crónica" 2). Estos acontecimientos sirvieron como plataforma para la Conferencia de las Naciones Unidas Contra el Racismo, la Discriminación y la Xenofobia -realizada en Durban, Sudáfrica, en 2001-, a la que asisten las hermanas Salgado (Espinosa, "Afrochilenos" 176). Este hecho histórico puso en tela de juicio los nacionalismos oficiales del país que se proclamaba racialmente inclusivo. Igualmente, resulta llamativo el hecho de que sean mujeres quienes representan la negritud chilena frente al mundo.

Ese mismo año, en Arica, las hermanas Salgado fundan Oro Negro, organización de gran importancia pues es el primer grupo que sostiene de manera pública la existencia de afrochilenos (Salgado, "Crónica” 3). Desde ahí comienzan a aparecer nuevas organizaciones: Lumbanga, Arica Negro, Colectivo de Mujeres Luanda, Hijas de Azapa, entre otras. En primera instancia, estas organizaciones se reúnen por parentesco y luego ponen atención a las características físicas e historias colectivas de un pasado que remite a una ascendencia africana. Posterior a esto, y una vez que el número de integrantes va creciendo, indagan en una materialidad cultural afro que se basa principalmente en la comida, bailes y costumbres religiosas, a través del rescate y la reconstrucción de su identidad. Todo esto se manifiesta en un movimiento de carácter histórico y sociopolítico sobre la problemática negra en el país y la lucha por el consecuente reconocimiento social, cultural y legal de las raíces africanas por parte del Estado ${ }^{16}$.

Hallazgos ETNOGRÁficos: ESPACiOS E INTELECTUALIdAdes AFROFEMENINAS

En este apartado se describirán diferentes papeles de las afrodescendientes en ámbitos sociales, culturales, políticos y económicos, en cuanto a la continuación de su cultura desde lo privado y lo público. En lo

16 Hasta el 2019, Chile no reconocía la presencia africana ni respondía frente a la ratificación del Convenio 169 de la OIT, realizada en 2008. 
privado, destacan sus roles en lo doméstico como madres, cuidadoras y dueñas de casa, donde se han ocupado de las familias, los hijos y la vida comunitaria, esto entendido más allá de los patrones patriarcales establecidos de crianzas (Reid Andrews 203). En África ancestral, la tradición oral forma parte integral en la vida de los africanos, donde las y los antiguos poseen y transmiten el saber, así se convierten en sabios de memoria y prestigio (Chávez 66). Según los relatos de los afrochilenos recopilados, las abuelas y madres cumplieron un rol en el proceso de reconstrucción y reivindicación identitaria, pues fueron ellas quienes transmitieron a sus descendientes la memoria que se explicita en enseñanzas de tradiciones y costumbres ${ }^{17}$, a pesar de la negación en la que se vieron inmersos. La mayoría de las y los colaboradores recuerdan cómo sus ancestras relataban eventos históricos, les enseñaban sobre percusión, baile y música, además de formas específicas de cocinar. Actualmente, la mayoría de las afrodescendientes llevan adelante una crianza consciente con pertenencia histórica racial e inculcan la negritud en sus sucesores.

Estos saberes no se restringen a las paredes de un hogar, pues las colaboradoras revelan que sus madres y abuelas también se encargaban del sustento económico. Ellas tenían un papel activo como trabajadoras campesinas y emprendedoras en la agricultura, comercio y artesanía en la región. Durante el siglo XX, un grupo de mujeres conocidas como las "comerciantas", realizaban transacciones comerciales y económicas de productos agropecuarios -frutas y verduras- que transportaban en angarillas $^{18}$ atadas en el lomo de los burros desde el valle de Azapa al Mercado San Marcos en Arica (Llerena y Salgado 295). Algunas participantes recuerdan a sus madres en estrecha relación con la tierra y el trabajo rural, las siembras y cosechas, así como también con la recolección de aceitunas; mientras que otras con la manufactura y creación de artesanías en base a totora, que después vendían en el mismo valle o en ferias de la ciudad. Desde aquí se desprende un ara de reconocimiento

17 Un elemento clave al hablar de una identidad afrodescendiente es la configuración familiar, pues la mayoría -tanto en la ciudad como en los valles- son unidas y extensas, y así generan una autoconciencia de pertenencia al grupo a través de lazos de parentesco. En la composición de la jerarquía familiar y en la configuración social de la comunidad, se puede sostener la idea de un linaje femenino debido a la relevancia de las ancestras.

18 Tipo de receptáculo grande. 
intelectual identitario en relación con la promoción y rescate de las tierras ocupadas durante siglos, su flora y fauna y su conexión con las mismas (Duconge y Guizardi 146; Cortés y Rivera 5).

En cuanto a la religiosidad, y en específico en lo recopilado en la celebración de la Cruz de Mayo - una fiesta religiosa traída por españoles y que ellos comenzaron a celebrar con detalles particulares-, desde antaño la mujer afrodescendiente se ha dedicado a arreglar y vestir a la cruz el día que se baja del cerro, junto con adornar el altar. El día en que se sube la cruz, se realiza una gran fiesta y son ellas quienes preparan los detalles de la comida -mondongo o picante de guatita ${ }^{19}$ - como de la misma festividad. Son de tal relevancia las figuras femeninas, que la mayoría de las cruces de las familias afro reciben el nombre de una ancestra $^{20}$. El culto a la cruz y los cerros tutelares se asocia a su vez a un homenaje a la pachamama (madre tierra), una figura femenina dadora de fertilidad y abundancia (Chávez 64). De esta manera, la Cruz de Mayo se posiciona como símbolo de la protección y culto de las antepasadas y a la herencia ancestral intelectual sobre las prácticas que dejaron, incluso varias de ellas son denominadas como "matriarcas"21.

Por otra parte, en la comunidad afrochilena hay múltiples aristas intelectuales fuera de la literatura convencional que giran en torno a la musicalidad de la oralidad, desde un aspecto de la escritura sonora del afro (Mendizábal 98). Con la conformación de comparsas y agrupaciones musicales como mecanismos de visibilización, irrumpieron en el ámbito público frente a la sociedad ariqueña y a lo largo de Chile. Estas formas de expresiones se asocian a lo intelectual y cultural representado por una exposición de elementos culturales materializados: el ritmo de tambores, lo carnavalesco, la creación de música y letras, la fabricación de instrumentos, el rescate de bailes propios, entre otros. Estas danzas

19 Comida que la comunidad afrodescendiente proclama como propia y que sostienen que las y los esclavizados cocinaban con los interiores y sobras que les daban sus patrones.

20 Dentro de las más reconocidas y antiguas del valle de Azapa: Cruz Julia Corvacho, Cruz de Mayo Eulogia Baluarte, Cruz de Mayo Flora Cardoso, Cruz de Mayo Ventura Baluarte, Cruz de Mayo Tomasa Baluarte o Cruz de Mayo Julia García.

21 El sistema matrilineal se sostiene por la idea de que la adscripción del sujeto con su linaje queda determinada por vía materna y sus principales familiares son parientes de su madre, así mismo las propiedades y las herencias de tierras provienen de la familia de la madre o de la abuela. 
representan simbólicamente situaciones de un pasado esclavista, como recoger algodón, la caña de azúcar y la recolección del olivo; actividades propias de aquel tiempo que teatralizan a través de una performance y que quedan plasmadas en las melodías y escritos. La principal danza es el "tumbe" o "tumba" (Letelier 2), que fue rescatado por medio de la recopilación de relatos de abuelas y abuelos del valle de Azapa, quienes rememoraban un estilo de danza realizado en periodo de fiesta o carnaval ${ }^{22}$. Hoy en día, este baile se ha masificado y se erige como característico de la comunidad afrochilena norteña, incluso está incluida como categoría en el Carnaval con la Fuerza del $\mathrm{Sol}^{23}$ y ha impulsado la instauración de un propio Carnaval Afro que se celebra desde el $2003^{24}$.

Las afrochilenas principalmente son bailarinas, mientras que los varones se encargan de tocar los instrumentos, aunque estos papeles no son estáticos ni privativos, ya que actualmente muchas de ellas tocan tambores e incluso hay grupos compuestos exclusivamente por mujeres, como Aluna Tambó. Las participantes establecen que los bailes -ya sean más tradicionales, como el tumbe, los caporales, el baile de morenos o los gitanos; o más nuevos, como el afromandingue- son una forma de visibilizar su cultura y que sienten como parte de su identidad. Sus danzas y vestimentas ${ }^{25}$ giran en torno a la manifestación política de sus dinámicas de identidades de raíces africanas, como distintivos de la comunidad y otros modos de resignificación: reivindicación negra, descosificación de los cuerpos y empoderamiento femenino (Chávez 125).

Las y los negros se reunían, percutían elementos caseros y trataban de botar a la pareja de baile por medio de un caderazo, cada vez que se festejaba se gritaba “itumbe!”, de ahí el origen del nombre.

23 El Carnaval Internacional Andino con la Fuerza del Sol Inti Ch'amampi Arica es una fiesta que se realiza cada año durante tres días en el verano.

24 Actualmente, se ha masificado la conformación de comparsas en otras regiones, como las de Coquimbo, Metropolitana, Valparaíso, Concepción, por mencionar algunas.

25 Dependiendo de la comparsa existen diferentes vestimentas, por ejemplo, algunas utilizan colores blancos pues asemeja la ropa que usaban las algodoneras históricas, otras usan colores vivos y cada día se expande el uso de turbantes africanos. 


\section{Política y MUJERES AFROCHILENAS}

Para analizar el rol participativo de las mujeres dentro de los movimientos afrodescendientes, daré cuenta de algunas lideresas, de las organizaciones en que participan y de algunas acciones que llevan adelante para entenderlas como productoras de determinados conocimientos, considerando que "el pensamiento afrolatinoamericano no ha sido monolítico como tampoco ha sido suficientemente reconocido en la mayoría de las reseñas sobre el pensamiento latinoamericano" (Guridy y Hooker 220). Estas mujeres han sido agentes de su propia historia, pues son principalmente ellas quienes han expuesto las prácticas de exclusión racial cuando el Estado nacional ha negado la existencia del racismo. En un primer momento, sus acciones constaron en rescatar una cultura y costumbres negras, que reivindican y demandan un reconocimiento social y cultural, desde la memoria y las múltiples manifestaciones de oralidad (De Friedemann 25; Mendizábal 100). Las afrochilenas poseen un gran compromiso con su labor como dirigentas de organizaciones y son quienes se hacen cargo de los intereses colectivos de su comunidad. Ello se refleja en que han alcanzado significativos logros y se proyectan en los mismos.

Según las y los colaboradores de esta investigación, la preponderancia femenina afro inició esta lucha en Arica, con las figuras de las hermanas Salgado, quienes fundaron el año 2001 la ONG Oro Negro ${ }^{26}$. Hasta la actualidad, las mujeres son quienes están a la cabeza como dirigentas de las múltiples organizaciones reivindicativas, donde llevan a cabo regularmente diferentes acciones políticas que realizan para organizarse y reclamar contra el Estado chileno y dar a conocer sus demandas; marchas, foros, seminarios y otras convocatorias.

En el valle de Azapa, en 2004 nace la ONG Lumbanga, de donde surge el año 2008 la primera organización exclusiva de mujeres afrochilenas: la Colectiva de Mujeres Luanda. Posteriormente, participan mujeres de la ciudad, quienes indagan en las incidencias políticas del tema de género y violencia y buscan romper con los estigmas asociados, la discriminación y degradación de las mujeres. Este grupo actualmente se autodenomina

26 En 2016 surgió el grupo de baile Piel de Ébano, perteneciente a Oro Negro. Su presidenta señala que este grupo tiene como objetivo visibilizar a la mujer $y$, en especial, a las más adultas, reivindicando su visibilización. 
como feministas asociadas principalmente a los feminismos negros, se reúne para trabajar temáticas de género y derechos humanos, por lo que se consolidan como una agrupación con capacidad de posicionar la temática desde la identidad de género afrodescendiente ${ }^{27}$. Estas mujeres afro plantean que por medio del conocimiento de su historia se podrán conocer a sí mismas y a su colectividad; conocimiento que ponen en práctica en la cotidianidad de sus vidas. Durante 2015, organizaron el Primer Congreso Internacional de Mujeres Afrodescendientes en Chile, el cual se realizó en Arica. Allí generaron un espacio colectivo para ellas, integraron la dimensión política e intelectual, debatieron sobre temáticas identitarias, de memoria, discriminación, derechos sexuales y reproductivos y sobre incidencia política, entre otros temas. En marzo de 2016, lanzaron un libro de memoria sobre este congreso, con el apoyo de la Universidad Santo Tomás y de la Fundación Heinrich Böll.

Hijas de Azapa es una organización que surge en 2012 y que tiene como fin rescatar las costumbres, tradiciones y religiosidades de las mujeres rurales del valle. El objetivo era capacitarse en áreas de emprendimientos orientados a la venta de comestibles y artesanías afro y cursos de talleres de formación política y violencia contra las mujeres, gestionados por entidades dependientes de la municipalidad como también de organismos privados. En el valle está la Red de Mujeres Rurales, Indígenas y Afrodescendientes de Azapa y Lluta, una sociedad civil creada en el año 2000. Si bien esta red no es netamente afro, se estima que un $40 \%$ de sus participantes sí lo son (Espinosa, Reconstrucción 144). También se destaca Arica Negro: Recuerdos de la Chimba, fundada en 2005 por las hermanas Lara, quienes la presiden actualmente. Esta organización se propone como objetivo el rescatar las tradiciones y las raíces costeras de afrodescendientes que se ubican en un sector de Arica conocido como la "Chimba", lugar antes habitado por pescadores afrodescendientes.

Un segundo momento estuvo marcado por el carácter político que adquirió el movimiento desenvolviéndose "hacia afuera", es decir, comenzando a propagarse por diferentes países. Este fue el periodo en

27 Según diferentes autoras -Lorde, Davis, Bell Hooks, entre otras-, la identidad de género en la cultura afrodescendiente ha sido investigada por el movimiento feminista negro, o Black Feminism, que busca llevar a cabo una lucha a nivel internacional frente al racismo y sexismo que existe sobre ellas, pues asumieron que ser mujeres de color, más que una definición biológica, es una categoría política. 
que se visibilizaron los temas del racismo y sexismo y sus consecuencias, que iniciaron procesos de denuncias junto a una formación educativa y de comunicación. Desde entonces, se da una articulación nacional y regional, en referencia al movimiento global que empezó a hacerlas partícipes. Por ejemplo, se acercan a la Red de Mujeres Afrolatinas, Afrocaribeńas y de la Diáspora ${ }^{28}$, un importante movimiento autónomo creado para su defensa, que posee incidencia política e intelectual a nivel internacional. Ellas impulsan estrategias y compromisos para hacer frente a temas como el racismo, el sexismo, la identidad y cultura, los derechos humanos, la participación económica y política, entre otros (Castro 74).

Desde el 2010, las mujeres afro de la región organizan una marcha para conmemorar masivamente el 25 de julio, correspondiente al Día Internacional de la Mujer Afrodescendiente, con el objetivo de dar a conocer sus voces y manifestarse por sus derechos. De este modo, se constituyen como sujetas activas en la lucha política que impactó en la conciencia crítica de activistas e intelectuales afrochilenas, "procesos además que en el ámbito académico dan lugar a los estudios postcoloniales, culturales y subalternos que colocan en el centro la construcción de los sujetos y las sujetas en contextos postcoloniales" (Curiel, "Descolonizando" 2). A raíz de estos planteamientos, se ha visibilizado el tema y surge dentro de este movimiento una política antisexista, antirracista y descolonizadora.

\section{INTELECTUALIDAD FEMENINA AFROARIQUEÑA Y AFROAZAPEÑA}

Por una parte, y según sus relatos, podemos ver que la confianza y autoestima de este grupo de mujeres fue creciendo en lo individual junto con el ser parte de un colectivo, fomentado por el autorreconocimiento. Esto dio pie a un primer momento marcado por la realización de encuentros donde ellas pudieran compartir experiencias, hablar sobre sus situaciones de vida, buscar apoyo y construir intelectualidades orales. Al mismo tiempo, intentaban recuperar algunas tradiciones y ancestralidades históricas.

Red internacional creada en 1992. Entre el 19 al 25 de julio del año 1992, se realizó el primer encuentro latinoamericano y caribeño de mujeres negras en República Dominicana, donde se crea la Red de Mujeres Afrolatinas, Afrocaribeñas y de la Diáspora. A esta reunión asistieron 350 mujeres de 32 países del continente, organizadas en grupos, colectivos y agrupaciones. 
Sin embargo, aún queda pendiente la tarea "de descubrir los espacios no tradicionales donde pensadores afrolatinoamericanos formularon sus aportes y de reimaginar creativamente el material de archivo disponible para descubrir las voces y experiencias perdidas" (Guridy y Hooker 258). De ese modo, el movimiento afrodescendiente femenino ha aportado en la constitución de figuras intelectuales y a su transformación social activa. Allí, sus historias particulares y políticas destacan desde el arte de la palabra no escrita o la "oralitura", que "reconoce la estética de la palabra plasmada en la historia oral” (De Friedemann 25).

Entre las afrochilenas del norte del país, también destaca un segundo momento basado en las producciones textuales; en especial: a) El capítulo "El legado africano en Chile" del libro de Sheila Walker, Conocimiento desde adentro (2010) ${ }^{29}$; el libro Afrochilenos: una historia oculta (2012), y los artículos "Crónica del Movimiento Pueblo Afrochileno: La lucha por el reconocimiento constitucional" (2015) y "Memorias marginadas del afrodescendiente chileno: la restitución de la identidad"30 (2019), todos escritos por Marta Salgado Henríquez; b) el artículo “iSoy Afroariqueña!” (2015), de Carolina Letelier Salgado; c) el libro Sentimientos de mi Tierra: Poemas (2016), escrito por María Elena Castillo ${ }^{31}$; d) el libro Identidad y Territorio. Afrodescendientes en Chile (2018), de Azeneth Báez ${ }^{32}$; e) el libro Desde las ancestras a la actualidad. Mujeres negras de Arica y sus resistencias (2019), gestionado por las miembras de la Colectiva Luanda y escrito por Carolina Cortés y Camila Rivera; y f) diversos artículos de opinión publicados en revistas y redes sociales ${ }^{33}$.

Estas obras, tal como expone Oliva (“Intelectuales" 51), están en relación con el proceso de autorreconocimiento y cobran vital importancia pues, en general, los escritos de los y las afrodescendientes tienden a ser marginados del mainstream de las instituciones académicas, literarias y

29 Ver el libro de Sheila S. Walker, Conocimiento desde adentro. Los afrosudamericanos hablan de sus pueblos y sus historias (2010), pp. 223-270, La Paz: Fundación Pedro Andavérez Peralta/Afrodiáspora Inc./Fundación Interamericana/Fundación PIEB.

30 Coescrito con Marco Llerena Rodríguez.

31 Afrodirigenta del valle de Azapa.

32 Coescrito con Cristián Báez.

33 Un ejemplo es el escrito de Milene Molina, "Nuestras ancestras y ancestros fueron esclavizados y traídos a este territorio hace 480 ańos: estamos acá antes del estado nación chileno" (2014). Disponible en https://www.revistarosa.cl/2020/12/21/ nuestras-ancestras-y-ancestros-fueron-esclavizados-y-traidos-a-este-territorio/ 
culturales del país. Se trata de "una inquietud intelectual que se nutre del movimiento social y organizativo [...] en el cual las mujeres no son una excepción [...] [y] se han posicionado políticamente en contra de la discriminación racial y de género" (Oliva, "Entre lo remoto" 182).

Es de importancia resaltar el ejercicio de sus autorías y el grado de autonomía de la voz autoral que poseen estos escritos, "pues sus textos facilitan seguir la pista de sus reflexiones, con sus continuidades y quiebres" (Oliva, "Intelectuales" 53), que también se ven en las tesis de licenciaturas y magister de estas mujeres. La tesis de Carolina Cortés en licenciatura en Historia aborda los estereotipos femeninos en la prensa escrita de 1909-1930, mientras que su tesis de magíster en Historia se denomina Mujeres negras en Arica colonial: Articulación y resistencia durante el siglo XVIII (UTA, 2020). La tesis de Derecho de Camila Rivera, junto a Carla Rubio, se titula Análisis crítico de los instrumentos de protección y su aplicación a las comunidades afrodescendientes en Chile (UTA, 2012). Estas mujeres proponen una mayor reflexión dentro del movimiento. Desde el autorreconocimiento de una identidad colectiva, analizan su historia de exclusión y actual realidad y generan discursos críticos de reparación histórica y visibilización de sus comunidades desde la academia, en un contexto donde las estructuras hegemónicas jerarquizan las diferencias culturales, por lo que asumen "una posición política en el espacio público" (Oliva, "Intelectuales" 55).

Siguiendo esta línea, las afrochilenas organizan constantemente charlas con intelectuales afrolatinas, a través de una Escuela de Autoformación Feminista Negra y Decolonial ${ }^{34}$-también llamada poscolonial-, donde realizan análisis interseccionales sobre temáticas de género y raciales tanto en los movimientos negros como en los feministas (Guridy y Hooker 248). De este modo, los feminismos negros latinos apuntan a nuevas vías para los pensamientos políticos del continente y son relevantes a la hora de analizar la participación de las mujeres en el campo intelectual, pues con el feminismo esta aumenta notoriamente.

34 Realizada durante 2019 en Arica. Han participado intelectuales afrolatinas como Ochy Curiel, Yurderyks Espinosa, entre otras. 


\section{REFLEXIONES FINALES: INTERSECCIONALIDAD E INTELECTUALIDAD}

En este artículo se mostró una mirada sobre género en la negritud afrochilena, mediante los papeles intelectuales que tienen las mujeres en su comunidad en el pasado y en el presente -ya sea en lo público como en lo privado, en lo político y en lo sociocultural-, los que en muchos casos se transforman en mecanismos de identificación, resistencias y luchas. Por medio del historizar sus experiencias y narrar sus memorias, se posicionan como autoras conscientes de su colectivo. Además, se establecen posicionamientos en el plano político al disputar las representaciones que se les han impuesto. Los primeros escritos de las afrochilenas se basan en recopilaciones de memorias de sus ancestros, mientras que las últimas publicaciones se respaldan en lo académico formal y así desarrollan análisis históricos, de carácter jurídico, entre otros. Estos eventos posicionan a estas mujeres como intelectuales afrodescendientes, ya que, desde sus organizaciones políticas y socioculturales, han reparado la exclusión de pensadores afrolatinoamericanos con un desarrollo propio, a través de la autoafirmación y reivindicación de sus propias identidades raciales y femeninas.

Además hemos constatado que las prácticas afrofemeninas han tenido agencia desde la esclavitud, cuando se comprometían en la resistencia cotidiana, como también en la incidencia dentro del ámbito jurídico por la manumisión de sus descendientes. Hasta el presente, las intelectuales afrochilenas representan una amplia diversidad de fuentes e ideas, como también de perspectivas políticas: de derecha, proyectos igualitarios, de izquierda y de feminismos negros. De este modo, la participación política y social de estas mujeres se orienta tanto a ámbitos generales de la comunidad afro como a ámbitos particulares de sus propias situaciones en torno al género. Estas luchas permitieron que se reconocieran como sujetas políticas líderes y como intelectuales, creadoras, cultoras, portadoras y transmisoras de conocimientos (Chávez y Araya 140).

Las mujeres afrodescendientes colaboradoras en esta investigación conservan sus dinámicas identitarias por medio de una reconstrucción de un estilo de vida basado en la figura de sus antepasados y la historia oral familiar, que, a su vez, transmiten a sus descendientes como una memoria genealógica. De este modo, cumplen los roles de forjadoras de nuevas generaciones junto al fortalecimiento de los núcleos, donde también 
se crean diálogos comunitarios. Esto se denota en aspectos culturales y religiosos, en las comparsas -a través de los bailes y vestimentas-, entre otros campos que forman parte de una "cultura afrochilena" propia, compartida entre la comunidad. En cuanto a lo económico, desde la chilenización, los relatos indican que las negras que permanecieron en el valle de Azapa cumplieron el papel de protectoras/encargadas de la contención de un quebrado núcleo familiar, junto con convertirse en sustentadoras económicas del hogar, ya sea en sus papeles como comerciantas y en sus trabajos en emprendimientos autogestionados.

Las afrochilenas han iniciado un camino de politización desde sus roles de madres, esposas e hijas, y ligan este perfil a su acción política, convirtiéndose en dirigentas activas. Dentro del debate entre lo privado y lo público, las colaboradoras poseen una mezcla de ambos mundos continuamente, debido a lo que amplían sus formas de participación en el campo político e intelectual, destacando el fuerte empoderamiento político femenino en la lucha por el reconocimiento. Desde el principio del proceso de etnogénesis, participaron en organizaciones comunitarias y luego, como principales lideresas del movimiento de emancipación, son sujetas activas en la transformación social. Las agrupaciones buscan tener una visibilidad política para concientizar su protesta y dar cuenta de la marginalización que sufren cotidianamente por ser mujeres y negras. Por ello, buscan una transformación social y así surge una política antisexista y antirracista, de la que incluso desarrollan actividades y escriben libros al respecto. Considerando esto último, se entiende que una de las características del pensamiento afrochileno haya sido la relación simbiótica entre lo cultural y lo político, en la cual las mujeres se convierten en activistas políticas con un alto poder de convocatoria. Por esto, se sostiene que es necesario volver la mirada al papel que juegan dentro del movimiento afro y del lugar que ocupa lo femenino en lo político.

En conclusión, se podría establecer que este grupo de mujeres afro se erigen como un pilar para el funcionamiento de su cultura, economía, política e intelectualidad oral y escrita. En un primer momento, se rescata la "oralitura" e historia de la literatura de la oralidad en diferentes espacios de experiencias de memoria, y luego se desarrollan desde lo académico tesis, artículos y libros al respecto. Ambos tipos de intelectualidades atesoran la tradición oral e incluyen preocupaciones políticas 
e intelectuales actuales, a través de diferentes experiencias culturales y literarias. Sin embargo, las investigaciones son escasas, por lo que las contribuciones intelectuales de mujeres afrochilenas continúan siendo doblemente invisibilizadas y ocupan una posición periférica en el campo intelectual. A pesar de ello, esta reflexión invita a explorar la condición intelectual que reposa en las dinámicas políticas y las agencias identitarias y comunitarias de las mujeres afrochilenas de Arica y el valle de Azapa.

\section{BiBLIOGRAFÍA}

Alarcón, Javiera, Araya, Isabel y Chávez, Nicole. Identidad Negra en tiempos de chilenización: memorias de abuelos y abuelas afrodescendientes de Arica y valle de Azapa. Santiago de Chile, Ministerio Culturas y Artes, 2017.

Araya, Isabel, Chávez, Nicole y Alarcón, Javiera. Cruz de Mayo Julia Corvacho; el legado negro del valle de Azapa. Santiago de Chile, Ministerio Culturas y Artes, 2019.

Arre, Montserrat y Paulina Barrenechea. "De la negación a la diversificación. Los intra y extramuros de los Estudios Afrochilenos". Tabula Rasa: revista de humanidades, № 27, 2017, pp. 129-160.

Artal, Nathalie. Á(f)rica: relatos y memorias afrodescendientes en Arica tras la chilenización y el conflicto entre Perú y Chile (1883-1929). Santiago de Chile, Universidad de Santiago, 2017.

Bengoa, José. La emergencia indígena en América Latina. México, Editorial FCE, 2004.

Briones, Viviana. "Mujeres Afrodescendientes en el corregimiento de Arica, siglo XVIII. De un silencio negro a la batalla por la libertad". Revista Ciencias Sociales, N 19, 2017, pp. 7-25.

Carneiro, Sueli. "Ennegrecer al Feminismo; Brasil". Ponencia Seminario La situación de la Mujer negra en América Latina, desde una perspectiva de género. 2001.

Castro, Evelyn. Representaciones contemporáneas de la esclavitud en las mujeres Afrocostarricenses, desde sus propias voces. Tesis para optar al grado de Magíster en estudios de Género y Cultura, Universidad de Chile, 2006. 
Campos, Luis. "Los negros no cuentan. Acerca de las demandas de reconocimiento de los afrodescendientes en Chile y La exclusión pigmentocrática”. Revista Antropologías del Sur, No 8, 2017, pp. 15-31.

Campos, Luis y María Paz Espinosa. Informe final Proyecto Recolección y clasificación de datos etnoterritoriales afrodescendientes de la Región de Arica y Parinacota. Mimeografiado, Santiago: Municipalidad de Arica-Oficina Afrodescendiente-UAHC, 2017

Chávez, Nicole. Mujeres afrodescendientes de Arica y el valle de Aza$p a$, raices africanas en el norte de Chile. Tesis para optar al grado de Licenciatura en Antropología, Santiago de Chile, Universidad Academia de Humanismo Cristiano, 2016.

Chávez, Nicole y araya, Isabel. Mujeres de Colores; historias femeninas afro andinas. Santiago de Chile, Ministerio Cultura y Artes, 2018.

Cortés, Carolina y Rivera, Camila. Desde las ancestras a la actualidad. Mujeres negras de Arica y sus resistencias. Arica, Ministerio Cultura y Artes, 2019.

Curiel, Ochy. "Los aportes de las afrodescendientes a la teoría y la práctica feminista. Desuniversalizando el sujeto 'Mujeres'”. Perfiles del Feminismo Iberoamericano, $\mathrm{N}^{\circ}$ 3, 2007, pp. 1-18.

. "Descolonizando el feminismo: Una perspectiva desde América latina y el Caribe". Primer Coloquio Latinoamericano sobre Praxis y Pensamiento Feminista realizado en Buenos Aires, 2009. "Género, raza, sexualidad. Debates contemporáneos". Intervención en Estudios Culturales, № 4, 2017, pp. 41-61.

Davis, Angela. Mujeres, raza y clase. Akal, Madrid, 2004.

De la Fuente, Alejandro y Reid Andrews, George. Estudios Afrolatinos: una introducción. Buenos Aires, CLACSO, 2018.

Duconge, Giselle y Menara, Lube Guizardi. "Afroariqueños configuraciones de un procesohistórico de presencia". Estudios atacameños, $\mathrm{N}^{\circ} 49,2014$, pp. 129-151.

Espinosa, María Paz. Reconstrucción identitaria de los Afrochilenos de Arica y el valle de Azapa. Tesis para optar al grado de Licenciatura en Antropología, Santiago de Chile, Universidad Academia de Humanismo Cristiano, 2013. 
"Afrochilenos en Arica: Identidad, organización y territorio". Antropologias del Sur, No3, 2015, pp. 175-190.

Fanon, Frantz. Los condenados de la tierra. México, FCE, 1963.

De Friedemann, Nina S. "De la tradición oral a la etnoliterarura". Revista América Negra, No 13, 1997, pp. 19-27.

Guridy, Frank y Hooker, Juliet. "Corrientes de Pensamientos sociopolítico afrolatinoamericano". Estudios Afrolatinos: una introducción, Alejandro De la Fuente y George Reid Andrews (eds), Buenos Aires, CLACSO, 2018, pp. 219-267.

Guber, R. Prácticas etnográficas. Ejercicios de reflexividad de antropólogas de campo. Buenos Aires, IDES/CAS, 2014.

Hammersley, Martyn y Atrinsons, Paul. Etnografía: Métodos de investigación. España, Editorial Paidós, 1994.

Hill Collins, Patricia. "La política del pensamiento feminista negro". ¿Qué son los estudios de mujeres?, Marysa Navarro (comp.), Buenos Aires, FCE, 1998, pp. 253-312.

Instituto Nacional de Estadísticas de Chile (INE). Encuesta de Caracterización Afrodescendiente. Santiago de Chile, 2013.

. Sintesis de resultados. Región de Arica y Parinacota. Censo 2017. Santiago de Chile, 2019.

Letelier, Carolina. “'Soy Afroariqueña!”. Rufián Revista, № 21, 2015, pp. 1-3.

Llerena, Marco y Marta Salgado. "Memorias marginadas del pueblo afrodescendiente chileno: la restitución de la identidad". Revista Anales, No 16, 2019, pp. 295-298.

Mellafe, Rolando. La introducción de la esclavitud negra en Chile. Tráfico y rutas. Santiago de Chile, Universidad de Chile, 1959.

MendizÁbal, Iván. "La lengua y lo afro: de la literatura oral a la oralitura (Ensayos)". Chasqui Revista Latinoamericana de Comunicación. Comunicación política y pueblos, $\mathrm{N}^{\circ}$ 120, 2012, pp. 93-102.

Oliva, Elena. "Entre lo remoto y lo foráneo: los afrodescendientes en Chile a propósito del libro Afrochilenos. Una historia oculta, de Marta Salgado". Meridional. Revista Chilena de Estudios Latinoamericanos, $\mathrm{N}^{\circ}$ 6, 2016, pp. 179-189. 
"Intelectuales afrodescendientes: apuntes para una genealogía en América Latina”. Revista Tabula, N²7, 2017, pp. 45-65.

Reid Andrews, George. "Afro-Latin America: Five Questions”. Latin American and Caribbean Ethnic Studies, N 4, 2009, pp. 191-210.

Salgado, Marta. Afrochilenos. Una historia oculta. Arica, Herco Editores S.A, 2013.

. "Crónica del Movimiento Pueblo Afrochileno: La lucha por el reconocimiento constitucional”. Rufián Revista, N²1, 2015, pp. 1-3.

Sото, Rosa. "Mujeres negras: sexualidad, enfermedad y salud en el Chile colonial". Revista Ciber Humanitatis, No 19, 2001, pp. 1-7.

Tijoux, María Elena. "El Otro inmigrante "negro" y el Nosotros chileno. Un lazo cotidiano pleno de significaciones". Boletín Onteaiken, $\mathrm{N}^{\circ} 17,2014$, pp. 1-15.

Vial Correa, Gonzalo. El africano en el Reino de Chile. Ensayo histórico-jurídico. Santiago de Chile, Instituto de Investigaciones Históricas PUC, 1957.

Vidal, Virginia. La Presencia Africana en Chile. Santiago de Chile, Editorial Poetas Antiimperialistas de América. 2008.

Wade, Peter. Raza y Etnicidad en Latinoamérica. Quito, Ediciones Abya-Yala, 2000.

Recepción: 30-09-20

Aceptación: 08-01-21 